\title{
TVA y TOA. Nuevas mallas ajustables de tensión en el tratamiento de la incontinencia urinaria de esfuerzo. Resultados preliminares
}

\author{
J. Romero Maroto, M. Ortiz Gorraiz, L. Prieto Chaparro, C. López López, J.M. Quílez Fenoll, \\ E. Rodríguez Fernández, J.J. Pacheco Bru
}

Servicio de Urología. Hospital Universitario San Juan de Alicante.

Actas Urol Esp 2006; 30 (2): 186-194

\section{RESUMEN}

TVA Y TOA. NUEVAS MALLAS AJUSTABLES DE TENSIÓN EN EL TRATAMIENTO DE LA INCONTINENCIA URINARIA DE ESFUERZO. RESULTADOS PRELIMINARES

Objetivos: Valoración de una nueva malla de incontinencia (TVA/TOA) que permite postoperatoriamente ajustar la tensión dada en quirófano.

Pacientes y Métodos: 62 pacientes tratados con la malla TVA, seguimiento medio de 14 meses (DS 7,8, rango 6-38). En 33 pacientes (53\%) se añadió corrección de algún prolapso. La valoración se ha realizado mediante historia clínica, exploración con vejiga llena con $250 \mathrm{cc}$ de suero salino, flujometría y residuo. 40 pacientes han rellenado 4 cuestionarios de calidad de vida. (QoL; ICIQ-SF; PGI-S y PGI-I).

Resultados: 42 pacientes eran continentes en la valoración post-operatoria. De estos, fue necesario disminuir la tensión en 7 (11\%) por obstrucción urinaria (flujo< $10 \mathrm{ml} / \mathrm{seg}$ y/o residuo). En 20 pacientes (32\%) se aumentó la tensión por continuar algún grado de incontinencia. Todos fueron dados de alta continentes y sin residuo. En la última revisión, 58 (93\%) son totalmente continentes y $4(6,5 \%)$ han mejorado notablemente su incontinencia. El $\mathrm{Q}_{\mathrm{MAX}}$ es 19,8 ml/s (DS 9,8). La urgencia miccional ha desaparecido o mejorado en $32(76 \%)$ de los pacientes que la presentaban preoperatoriamente y ha aparecido en 3 (15\%) de los que no la presentaban. La historia clínica muestra un alto grado de concordancia con el cuestionario ICIQ-SF $(K a p p a=0,89)$ en cuanto a incontinencia de esfuerzo se refiere, disminuyendo ostensiblemente $(K a p p a=0,13)$ cuando se toma en consideración la incontinencia por urgencia. De los 40 pacientes que completaron los cuestionarios de calidad de vida, 34 (85\%) tienen una puntuación superior a 95 sobre 110 en el QoL. 30 (75\%) tiene una puntuación inferior a 6 en ICIQ-SF. 32 (80\%) tienen una percepción de normalidad y $4(10 \%)$ de enfermedad leve en el PGI-S. En el PGI-I $29(72,5 \%)$ están mucho mejor y $11(27,5 \%)$ bastante mejor. Existe relación directa entre urgencia miccional y pérdida de calidad de vida.

Conclusión: La malla TVA (transvaginal ajustable) permite ajustar la tensión dada en quirófano, permitiendo corregir los defectos y los excesos.

Palabras clave: Incontinencia urinaria. Cirugía. Sling ajustable. Cuestionarios. Calidad de vida. Flujo máximo.

\section{ABSTRACT \\ TVA AND TOA. NEW ADJUSTABLE MESH FOR THE TREATMENT OF FEMALE STRESS INCONTINENCE. PRELIMINARIES RESULTED}

Objectives: Evaluation of a new mesh for incontinence (TVA/TOA) which enables the degree of tension applied during surgery to be readjusted at the post-operative stage.

Patients and Method: 62 patients treated with the TVA mesh and monitored over a 14-month period (SD 7.8, range 6-38). In 33 patients (53\%) some other pelvic prolapse was corrected. Evaluation was carried out by clinical report, examination of bladder full of 250 saline solution, flowmetry and urinary residue. 40 patients filled in 4 quality of life questionnaires (GoL; ICIQ-SF; PGI-S; PGI-I).

Results: 42 patients were found to be objectively continent in the post-operative evaluation. Of these, it was necessary to reduce tension in 7 cases (11\%) due to urinary obstruction (flow< $10 \mathrm{ml} / \mathrm{sec}$ and/or residue). The tension of the mesh was tightened in 20 patients $(32 \%)$ due to continue with a certain degree of incontinence. All patients were discharged as continent and with no residue. In the last revision, 58 patients (93\%) proved to be objectively continent and 4 (6.5\%) showed a notable improvement in their incontinence. The $\mathrm{Q}_{\mathrm{MAX}}$ is $19.8 \mathrm{ml} / \mathrm{sec}$ (SD 9.8). The mictional urgency had disappeared or improved in 32 of the patients who had this prior to operation (76\%) and had appeared in 3 of the patients who didn't (15\%). The clinical report showed a high level of consensus with the ICIQ-SF survey $(K a p p a=0.89)$ regarding stress incontinence, diminishing clearly $(K a p p a=$ 0.13 ) when urge incontinence was taken into account. 34 (85\%) patients scored over 95 out of 110 in the $\mathrm{QoL}$. 30 (75\%) scored less than 6 in ICIQ-SF. 32 (80\%) showed a perception of normality and 4 (10\%) slight illness in the PGI-S. In the PGI-I 29 (72.5\%) were much better and $11(27.5 \%)$ quite a lot better. A relation exists between urgency and dismissed quality of life.

Conclusion: With the TVA (trans-vaginal adjustable) mesh it is possible to adjust the tension originally applied during surgery at the post-operative stage, so that any defects or excesses can be corrected.

Keywords: Urinary incontinence. Surgery. Adjustable sling. Questionnaire. Quality of life. Maxim flow. 
La banda suburetral libre de tensión (TVT), introducida por Ulmsten en 1995, consigue aproximadamente un $90 \%$ de éxito, mantenido en el tiempo, en la incontinencia de esfuerzo femenina ${ }^{1-4}$. Sin embargo, ocasiona disfunción miccional de llenado y vaciado en un porcentaje considerable de $\operatorname{casos}^{5-7}$.

La técnica TOT, basada en el mismo concepto libre de tensión, aparte de obviar la realización de cistoscopia, intenta disminuir el componente obstructivo del TVT. Los resultados preliminares no parecen confirmar plenamente esta idea ${ }^{8,9}$.

Parece, por tanto, que si la tensión dada no es suficiente continúa la incontinencia y si la tensión es excesiva se origina obstrucción urinaria. En este sentido, la técnica debería ser de tensión adecuada y el problema radica precisamente en dar en el campo quirúrgico dicha tensión adecuada.

Presentamos el resultado de una banda suburetral, transvaginal y transobturador (TVA, TOA), que permite ajustar la tensión hasta varios días después de la intervención quirúrgica, permitiendo así corregir los errores por defecto o por exceso cometidos en la cirugía.

\section{MÉTODOS}

80 casos han sido intervenidos en nuestro servicio, 70 con TVA y 10 con TOA, desde enero 2002 hasta enero 2005. De éstos, 62 casos TVA tienen una evolución superior a 6 meses y son el motivo de esta comunicación.

Todos los pacientes fueron evaluados con historia clínica, exploración física, flujometría y residuo postmiccional. La incontinencia se valoró con la vejiga llena con $250 \mathrm{ml}$ de suero salino, haciendo toser a la paciente en decúbito y bipedestación.

Las Tablas 1 y 2 muestran las características preoperatorias de las pacientes. La incontinencia se clasificó en grave, moderada y leve teniendo en cuenta si se producía a mínimos, moderados o grandes esfuerzos y si en la exploración física se producía una fuga importante con un solo golpe de tos, tres golpes de tos o más golpes de tos.

La bandeleta TVA esta hecha con una malla de polipropileno monofilamento macroporo. El modelo definitivo lleva insertados dos grupos de hilos también de polipropileno (Fig. 1).
Tabla 1

Características preoperatorias de las pacientes (I)

\begin{tabular}{lcc}
\hline Caracteristicas & media \pm DS & rango \\
\hline Edad (años) & $58 \pm 10,8$ & $28-79$ \\
Tiempo de seguimiento & $14 \pm 7,8$ & $6-38$ \\
Flujo máximo (ml/s) & $25,6 \pm 12,7$ & $5-62$ \\
Volumen miccional (ml) & $280 \pm 138$ & $160-695$ \\
Tiempo de flujo (s) & $26 \pm 12,8$ & $9-60$ \\
Residuo postmiccional (ml) & $200 \pm 72$ & $100-300$ \\
[6 casos] & & \\
\hline
\end{tabular}

\section{Tabla 2}

Características preoperatorias de las pacientes (II)

\begin{tabular}{|c|c|c|c|}
\hline Caracteristicas & \multicolumn{3}{|c|}{$\mathbf{n}^{\circ}$ pacientes - \% } \\
\hline \multicolumn{4}{|c|}{ Grado incontinencia } \\
\hline Grave & & 34 (55\%) & \\
\hline Moderada & & 22 (35\%) & \\
\hline Leve & & $6(10 \%)$ & \\
\hline Urgencia & & $42(67 \%)$ & \\
\hline \multicolumn{4}{|l|}{ Cirugia previa } \\
\hline Incont. esfuerzo & & $4(6,5 \%)$ & \\
\hline Histerectomía & & $11(17 \%)$ & \\
\hline Cistocele & & $4(6,5 \%)$ & \\
\hline Prolapso & Grado I & Grado II & Grado III \\
\hline Cistocele & $20(32 \%)$ & 12 (19\%) & $12(19 \%)$ \\
\hline Rectocele & $20(32 \%)$ & $2(3 \%)$ & $2(3 \%)$ \\
\hline Uterino & $9(14 \%)$ & $4(6 \%)$ & $3(4 \%)$ \\
\hline Cúpula & - & $3(4 \%)$ & $1(2 \%)$ \\
\hline
\end{tabular}

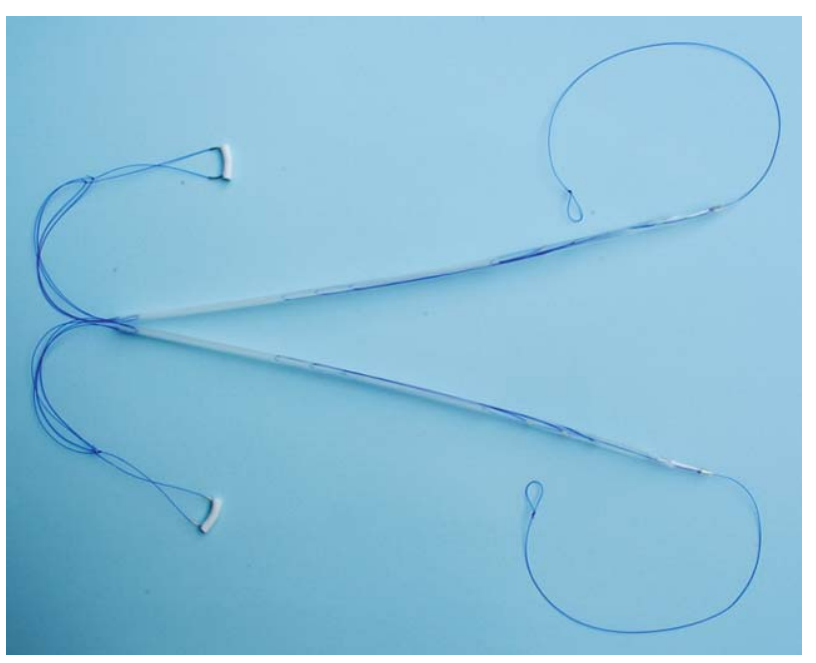

FIGURA 1

El primero formado por dos hilos situados lateralmente a un centímetro y medio de la línea media de la bandeleta, que serán exteriorizados a través de cara anterior vaginal y que servirán para destensar. El segundo, formado por tres 
hilos en cada rama de la bandeleta, está situado a distinta distancia de la línea media. Serán exteriorizados por donde sale la malla al exterior y permitirán tensar dicha malla (Fig. 2).

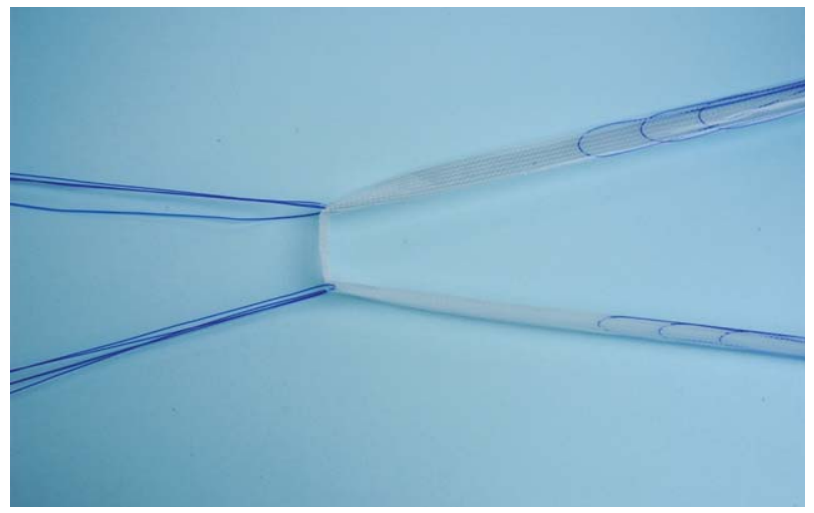

\section{FIGURA 2}

La bandeleta TVA la situamos, a través de una pequeña incisión, en pared vaginal anterior, en uretra proximal, dejándola muy holgada, prácticamente sin tensión. La malla ha sido pasada indistintamente en 50 ocasiones de hipogastrio a vagina y en 12 de vagina a hipogastrio, sin valorar dicha variable. Una vez colocada la malla, se extrae la vaina envolvente de plástico, se exteriorizan a cada lado de la incisión vaginal los hilos inferiores y se cierra la pared vaginal (Fig. 3). A continuación, se corta la malla sobrante en hipogastrio y dependiendo de la distancia de la uretra a piel en hipogastrio, se cortan también uno, dos o ninguno de los hilos laterales superiores (Fig. 4). Dejamos siempre un tapón vaginal de gasa empapado en povidona yodada.

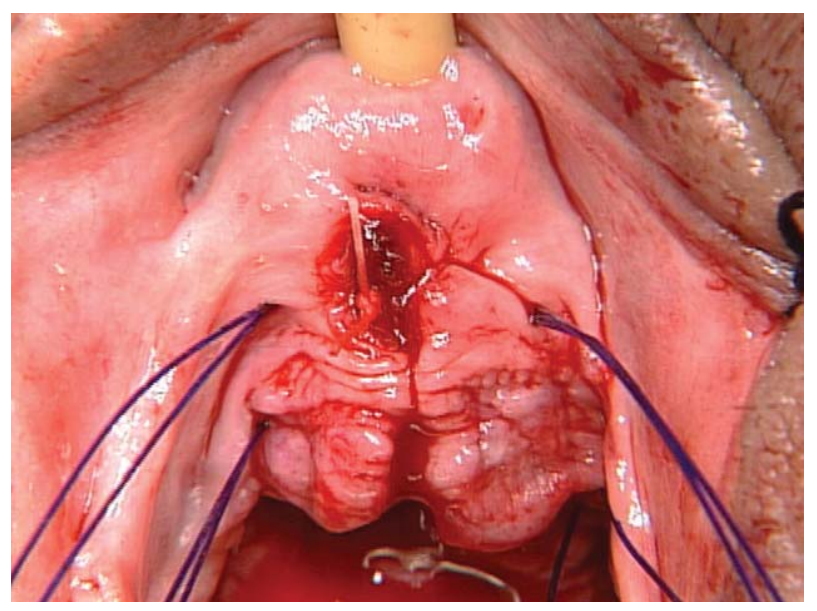

FIGURA 3

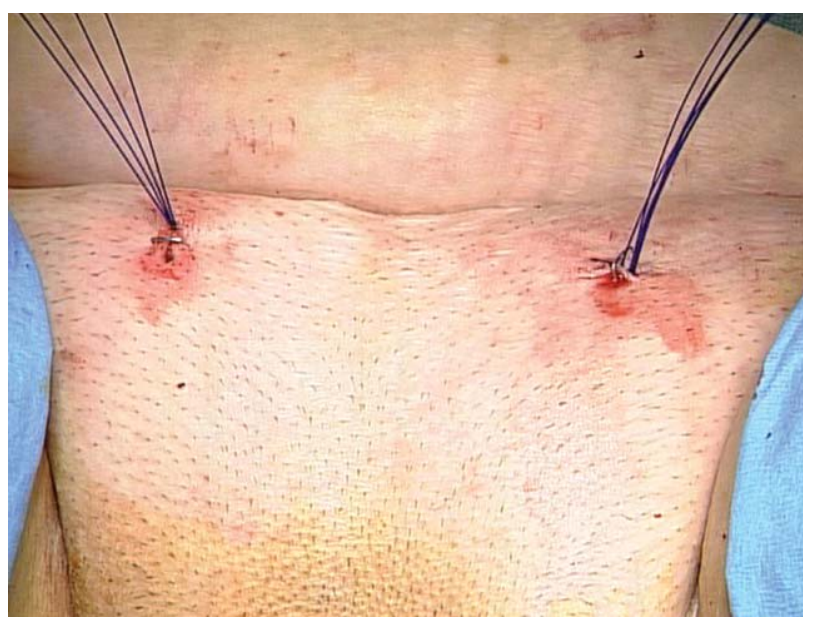

FIGURA 4

En 29 pacientes (47\%) se ha realizado solamente implantación de TVA. En 33 (53\%) se ha realizado simultáneamente otra cirugía. Se hicieron 7 histerectomías, 14 implantaciones de malla anterior, 7 plastias anteriores, 4 plastias posteriores y 5 pexias sacras.

52 intervenciones han sido hechas con anestesia intradural y 10 con anestesia general. En todos los casos dejamos talla mínima vesical.

\section{Valoración postquirúrgica}

Al día siguiente de la cirugía o más tarde si la situación de la paciente no lo permite (el ajuste más tardío se ha realizado a los 5 días en un caso de cirugía simultánea con pexia sacra) valoramos la situación miccional:

1. Llenamos la vejiga a través de la talla mínima con $250 \mathrm{ml}$ de suero salino.

2. Hacemos toser a la paciente en decúbito, bipedestación y andando.

Si existen fugas, infiltramos con anestesia local y tiramos de uno de los hilos suprapúbicos aproximadamente $0,5 \mathrm{~cm}$. Volvemos a repetir la exploración y así sucesivamente hasta que comprobamos la continencia en las tres situaciones, decúbito, bipedestación y andando. Realizamos, entonces:

3. Flujometría y medición de residuo postmiccional.

Si el flujo máximo es inferior a $10 \mathrm{ml} / \mathrm{s}$ o si existe residuo superior a $50 \mathrm{ml}$ destensamos la malla, tirando de uno de los hilos vaginales aproximadamente $0,5 \mathrm{~cm}$, comprobamos la continencia y hacemos, de nuevo, flujometría y medición de residuo. 
Cuando la paciente es continente en todas las situaciones, tiene un flujo máximo superior a $10 \mathrm{ml} / \mathrm{s}$ y no existe residuo, se cortan y se extraen los hilos, se retira la talla vesical y la paciente es dada de alta si su situación lo permite.

\section{Valoración resultados}

Todas las pacientes fueron valoradas con historia clínica, exploración física, flujometría y medición de residuo cada seis meses el primer año y una vez al año posteriormente. La incontinencia se valoró llenando la vejiga con $250 \mathrm{ml}$ con suero salino y pidiendo a la paciente que tosa y realice Valsalva en decúbito y bipedestación.

Se administraron cuatro cuestionarios validados a 40 pacientes ${ }^{10-13}$ :

1. Impresión global de severidad de la enfermedad del paciente (PGI-S).

2. Calidad de vida en la incontinencia (QoL).

3. Forma abreviada ICS/OMS (ICIQ-SF).

4. Impresión global de mejoría del paciente (PGI-I).

Mediante el paquete informático SPSS 12.0 para Windows de Microsoft ${ }^{\mathrm{TM}}$ se llevó a cabo el análisis estadístico descriptivo de la serie, con cálculo de medias, desviación estándar y rango. Igualmente se compararon mediante estadístico t-Student para flujo máximo y $\chi^{2}$ para número de ajustes, los diferentes grupos de pacientes. Se valoró también el grado de concordancia entre la historia clínica y los cuestionarios administrados, así como el índice de acuerdo entre cuestionarios, mediante la prueba Kappa. Finalmente se realizó análisis univariante mediante coeficiente de correlación de Spearman para ver posibles asociaciones entre los diferentes factores. No se realiza regresión logística multivariante debido al gran número de variables independientes analizadas lo que hace necesario un tamaño muestral mucho más amplio para tener significación los resultados.

\section{RESULTADOS}

La estancia media ha sido de dos dias $(2 \pm 0,3)$ en los casos de implantación simple de TVA y de cinco días $(5 \pm 1,6)$ en aquellos otros en los que se realizó otra cirugía añadida. No ha habido ninguna infección ni erosiones vaginales o uretrales y no ha sido necesario retirar ninguna malla. Tampoco ha existido hemorragia que necesite transfusión.

42 pacientes (68\%) eran continentes en la valoración inicial postcirugía. De estos, 7 (17\%) mostraban un flujo inferior a $10 \mathrm{ml} / \mathrm{s}$ y residuo superior a $150 \mathrm{ml}$. Igualmente 20 (32\%) mostraban un grado mayor o menor de incontinencia.

La tensión fue ajustada en 27 (44\%) pacientes; en 20 (32\%) pacientes incontinentes se aumentó la tensión y en 7 (11\%) obstruidos fue disminuida. 13 pacientes necesitaron, en la misma sesión, más de un ajuste $(2,5 \pm 0,7$, rango $2-4)$, requiriendo un mayor número de ajustes aquellos casos en los que se asoció cirugía de prolapso $(2,7 \pm 0,7$, rango 2-4) que en los que solo se implantó TVA $(2,3 \pm 0,8$, rango $2-4)$ sin significación estadística $(p=0,45)$. Al final del ajuste las 62 pacientes eran continentes, 55 tenian un flujo superior a 10 $\mathrm{ml} / \mathrm{s}$ y no tenían residuo. 7 tenían el flujo máximo inferior a $10 \mathrm{ml} / \mathrm{seg}(7,7 \pm 0,9$, rango $7-9$ $\mathrm{ml} / \mathrm{s}), 6$ sin residuo y 1 de ellos con un residuo superior al 25\% del volumen miccional. De estos siete, 4 correspondian al grupo que tras la cirugía estaba obstruido y 3 al grupo que había permanecido incontinente (Fig. 5).

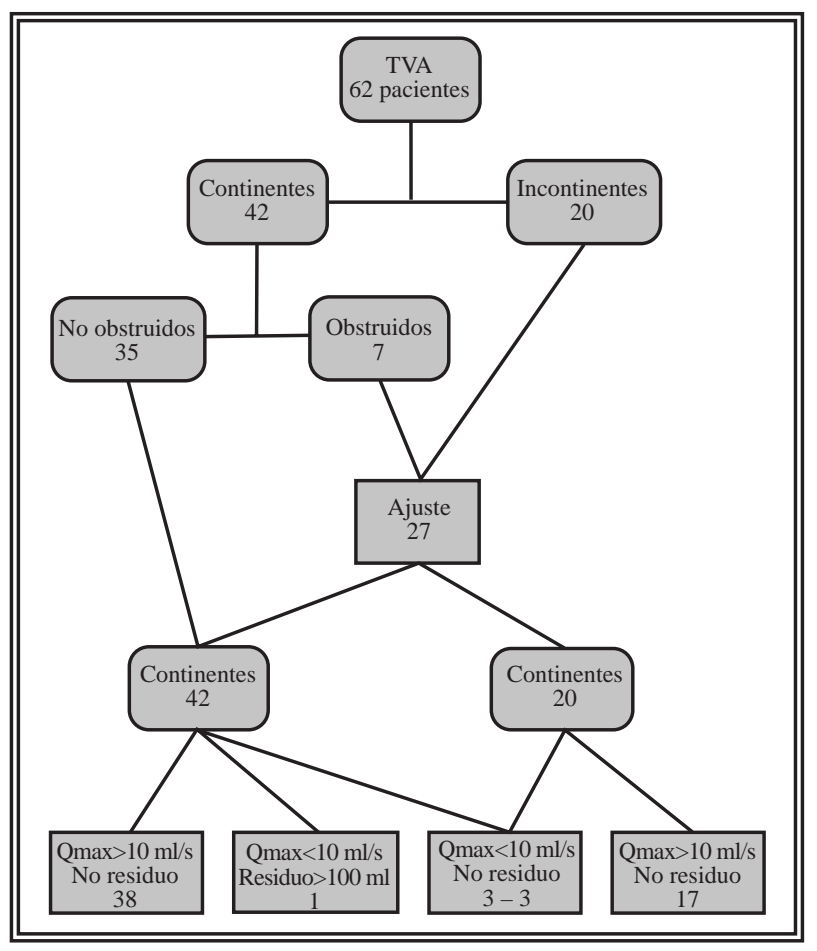

FIGURA 5. Evolución de las pacientes en función de su situación tras la cirugía. 
No hubo relación entre cirugía simple o asociada con prolapso y la necesidad de ajuste $\left(\chi^{2} d e\right.$ Pearson 0,86, sig. asint. 0,35).

En la última revisión realizada $(14 \pm 7,8$ meses, rango 6-38) y según lo recogido en la historia clínica, $58(93,5 \%)$ pacientes son continentes y 4 $(6,5 \%)$ han mejorado su incontinencia considerablemente. 37 (60 \%) no refieren urgencia miccional y 25 (40\%) la presentan. De los 42 que presentaban urgencia preoperatoriamente, ha desaparecido en 20 (48\%) y ha disminuido de intensidad en 12 (28\%), manteniéndose igual en $6(14 \%)$ casos y empeorando en 4 (10\%). De los 20 que preoperatoriamente no la presentaban ha aparecido de novo en 3 casos (15\%).

El flujo máximo medio en el seguimiento fue $19,8 \mathrm{ml} / \mathrm{s}$, (DS 9,8, rango 6-55), significativamente superior $(\mathrm{p}=0,001)$ que el correspondiente al alta de las pacientes $(14,6 \pm 5,5$, rango $7-35)$. Una paciente que no presentaba residuo al alta lo mostró en el seguimiento, oscilando entre $40 \mathrm{cc} \mathrm{y}$ $100 \mathrm{cc}$, no necesitando autosondajes. Por el contrario, el residuo desapareció en la paciente que lo tenía en el momento del alta.

Cuando comparamos el flujo máximo tras la última revisión en los pacientes con urgencia y sin urgencia (Fig. 6) no encontramos diferencia significativa $(p=0,10)$. Cuando la comparación se

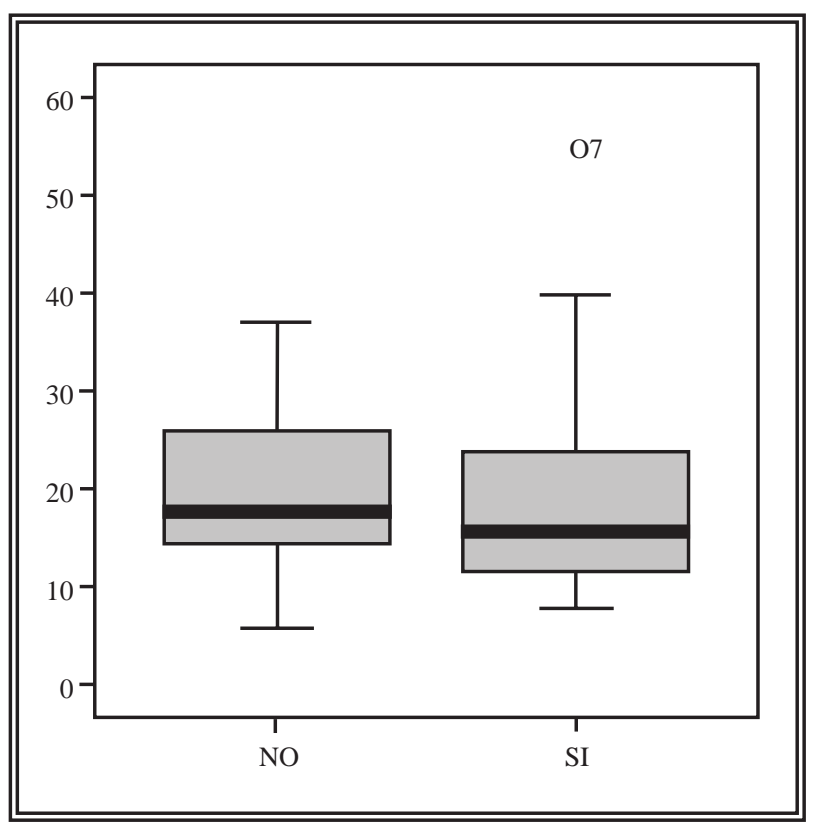

FIGURA 6. Comparación del Qmax según presencia o no de urgencia tras la cirugia. hizo entre aquellas pacientes intervenidas sólo de incontinencia con respecto al grupo de incontinencia más prolapso (Fig. 7) tampoco existió diferencia significativa $(\mathrm{p}=0,10)$.

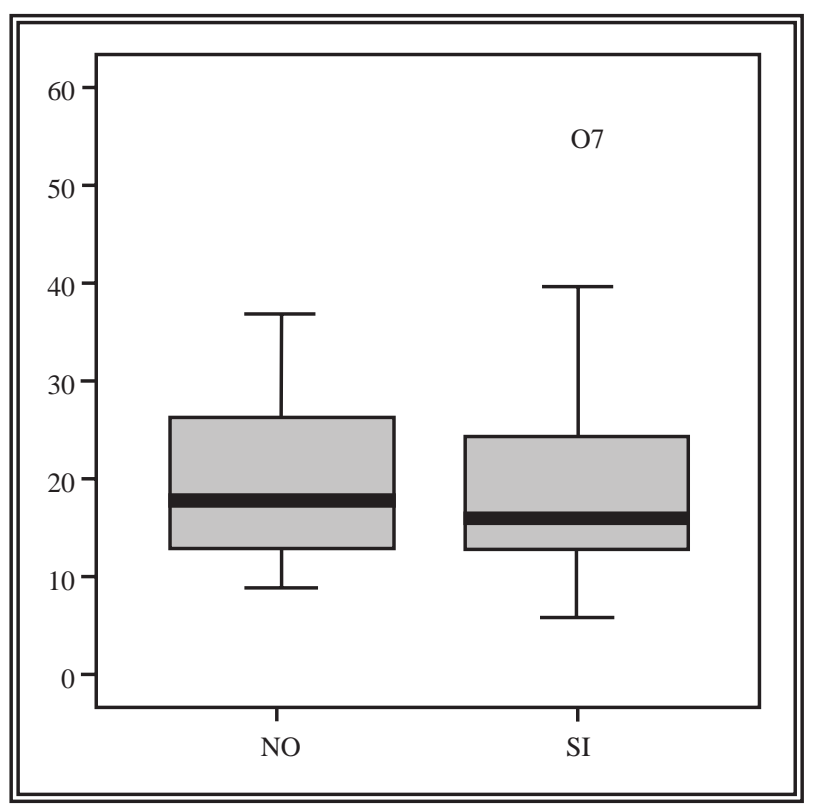

FIGURA 7. Comparación del Gmax según la asociación o no de cirugía del prolapso.

La Tabla 3 muestra el resultado de los cuatro cuestionarios administrados a 40 pacientes.

Se valoró el grado de concordancia entre los resultados de la historia clínica y el resultado del cuestionario ICIQ-SF, encontrándose un alto indice de acuerdo en cuanto se refiere a la incontinencia urinaria de esfuerzo (Kappa=0,89, pvalor $=0,000$ ), reduciéndose estas cifras cuando se tomó en cuenta también la incontinencia de urgencia (Kappa=0,13, p-valor=0,20).

Tabla 3

Resultado de los cuatro cuestionarios tras la intervención

\begin{tabular}{lcccc}
\hline \multicolumn{4}{l}{ Cuestionario } & \multicolumn{3}{c}{ Resultados } \\
\hline BoL & $95-110$ & $80-94$ & $60-79$ & $<59$ \\
& 34 & 0 & 4 & 5 \\
PGI-S & normal & leve & moderado & grave \\
& 32 & 4 & 1 & 3 \\
ICIQ-SF & 2 & $3-6$ & $7-11$ & $12-20$ \\
& 20 & 10 & 5 & 5 \\
PGI-I & mucho & bastante & un poco & igual o peor \\
& mejor & mejor & mejor & \\
& 29 & 11 & 0 & 0 \\
\hline
\end{tabular}


La Tabla 4 muestra la concordancia existente entre los cuestionarios de calidad de vida, tomando como buena calidad de vida las contestaciones normal y leve, menos de 6 puntos, más de 95 puntos y mucho mejor - bastante mejor en los cuestionarios PGI-S, ICIQ-SF, QoL y PGI-I respectivamente.

Tabla 4

Grado de acuerdo entre distintos cuestionarios

\begin{tabular}{lcc}
\hline Cuestionarios & Índice Kappa & p-valor \\
\hline PGI-S y GoL & 0,47 & $<0,001$ \\
PGI-S y ICIQ-SF & 0,50 & $<0,001$ \\
ICIQ-SF y GoL & 0,77 & $<0,000$ \\
\hline
\end{tabular}

El análisis univariante demuestra una buena correlación, coeficiente de correlación de Spearman de 0,8, entre la urgencia miccional previa y la urgencia miccional postoperatoria. Hay una correlación ligera, coeficiente 0,376 , entre calidad de vida y urgencia miccional postoperatoria. Ni la edad, ni la cirugía simultánea del prolapso han influido en la calidad de vida postoperatoria, coeficiente 0,084 y 0,089 respectivamente.

\section{DISCUSIÓN}

La banda libre de tensión (TVT) corrige la incontinencia de esfuerzo en un porcentaje variable entre $67 \%$ y $95 \% 14,15$, manteniéndose dicha curación en el tiempo. Como efecto secundario puede producirse una disfunción miccional que llega hasta el 60\% en algunas series ${ }^{6}$. Nuestra intención al diseñar la banda ajustable de tensión (TVA) ha sido poder corregir tras la cirugía la tensión dada en el quirófano, para intentar mejorar los porcentajes de continencia evitando los efectos obstructivos.

La continencia en el postoperatorio inmediato, previa al ajuste, ha sido del 68\%, rango inferior de lo publicado en la literatura y también de lo obtenido por nosotros en series anteriores ${ }^{2}$. La causa ha sido la mínima tensión dada a la malla en la cirugía, motivado esto porque, en el ajuste, la maniobra de tensar es mas sencilla que la de destensar. A pesar de la mínima tensión dada, 7 de los casos continentes no han cumplido nuestro criterio de no obstrucción y hemos tenido que disminuir la tensión previamente dada, lo que muestra la dificultad de calcular la tensión en quirófano.

Las situaciones de continencia sin obstrucción, continencia con obstrucción o incontinencia han dependido en muchos casos de solamente 0,4-0,5 cm de arrastre de la malla. Esto ha hecho que en ocasiones hayamos tenido que tensar y destensar varias veces la malla y pone, de nuevo, de manifiesto la dificultad de dar en quirófano la tensión adecuada.

En 7 casos no ha sido posible conseguir un flujo superior a $10 \mathrm{ml} / \mathrm{s}$. Una minima disminución de tensión suponía pasar a la situación de incontinencia. Se trataba de pacientes con un flujo máximo bajo preoperatoriamente. Bumsik et al. ${ }^{16}$ encontraron como único factor predictivo de retención urinaria post TVT la disminución de flujo máximo. Millar et al. ${ }^{17}$, por el contrario, no encontraron asociación entre el flujo previo y la retención urinaria posterior. Sí que existía con la ausencia de contracción del detrusor durante la micción. Es posible que tanto el flujo disminuido como la ausencia de contracción detrusoriana durante la micción traduzcan una cierta hipoactividad vesical y que la disminuida contracción detrusoriana postoperatoria no sea capaz de vencer la mínima tensión dada a la malla, ocasionando obstrucción y retención urinaria. De ahí la dificultad de encontrar el punto, que permitiendo el vaciado vesical, corrija, a su vez, la incontinencia.

Todas las pacientes fueron dadas de alta continentes y ninguna necesitó sonda vesical o autosondajes.

$\mathrm{El}$ ajuste pudo realizarse tanto en pacientes a quienes se había operado sólo de incontinencia como en aquellas otras a quienes se había realizado también corrección de algún tipo de prolapso. El número de maniobras fue superior en el segundo grupo aunque sin significación estadística. El ajuste lo hicimos infiltrando con anestesia local el trayecto de la malla. No lo hicimos en los primeros casos pero se producían ciertas molestias. Es más sencillo tensar que destensar por lo que siempre procuramos dejar la malla realmente sin tensión. Para destensar es necesario ejercer una tracción considerable. 
La continencia de esfuerzo, valorada con la vejiga llena con $250 \mathrm{ml}$ en decúbito y bipedestación, con Valsalva y tosiendo, se ha mantenido en $58(93 \%)$ pacientes y en $4(7 \%)$ ha mejorado substancialmente. Estos resultados, con un seguimiento medio de 14 meses, son claramente competitivos con los publicados en la literatura.

El flujo máximo en el seguimiento es superior, con significación estadística, respecto al obtenido en el postoperatorio inmediato. No sabemos si la resolución de un posible edema postoperatorio puede explicar esto. Posiblemente pueda haber existido también un ligero descenso de la malla implantada. En cualquier caso, no parece que exista retracción de la misma o esta ha sido compensada por las otras causas.

La urgencia miccional ha desaparecido o mejorado en el 76\% de los casos donde existía previamente. Parecería así que la hipermovilidad uretral, posiblemente por el paso de orina a uretra, es la causante de la misma. La existencia de prolapsos es otra causa que la origina, desapareciendo al corregir los mismos en más del $60 \%$ de los $\operatorname{casos}^{2,18}$. En nuestras pacientes coincidían la incontinencia con urgencia y el prolapso en 23 casos, habiendo desaparecido o disminuido en 14 (60\%) ocasiones.

La obstrucción urinaria causada por la cirugía es otra de las causas de aparición o empeoramiento de la urgencia existente, mejorando al solucionar la misma ${ }^{19}$. En nuestros casos no ha existido diferencia significativa en el flujo máximo entre el grupo donde ha persistido la misma y en el que ha desaparecido o disminuido.

Urgencia miccional ha aparecido en 3 casos de los 20 donde no existía previamente (15\%), cifras similares a las comunicadas por otros autores ${ }^{20}$. En estos 3 casos el flujo máximo fue $32 \pm 21 \mathrm{ml} / \mathrm{s}$, rango 11-55, flujo que junto a la ausencia de residuo urinario elimina también, en principio, el diagnóstico de obstrucción. Deben existir otras causas, aparte de la obstrucción, posiblemente lesiones de ramas nerviosas o reacciones inflamatorias a la malla, que colaboren o causen en ocasiones la aparición de urgencia miccional.

La banda la hemos situado en uretra proximal en vez de en uretra media. Nuestros resultados son totalmente comparables, tanto en porcentajes de continencia como en desaparición de urgencia o urgencia de novo a las series publicadas con la técnica TVT. Kaum y Wolf ${ }^{21}$ marcando la malla de Prolene con hilos radio-opacos encuentran los mismos resultados en aquellos casos en los que la malla está en uretra media que en aquellos donde la malla se ha desplazado a uretra proximal. La tensión dada a la malla aparece así como elemento más importante que su localización media o proximal en cuanto a porcentaje de éxitos y complicaciones se refiere y pone en duda que la localización en uretra media y el efecto dinámico de angulamiento de la misma sea la causa de la continencia postoperatoria $^{22}$.

Los resultados recogidos en el cuestionario administrado reducido ICIQ-SF, que define existencia de incontinencia y tipo de la misma, tiene una buena concordancia con lo recogido en la historia clínica en cuanto se refiere a incontinencia de esfuerzo (Kappa $=0,89)$. La concordancia es menor (Kappa $=0,13$ ) cuando no se separan los tipos de incontinencia. Rodríguez y $\operatorname{Raz}^{23}$ encontraron discrepancias entre los resultados clínicos y un cuestionario autoadministrado, y Haab et al. ${ }^{24}$ hallaron que esto es más acentuado cuando se tiene en cuenta la incontinencia por urgencia. Chaikin et al. ${ }^{25}$ encontraron, por el contrario, una buena concordancia entre la historia, utilizando un diario miccional, y los cuestionarios administrados cuando se utilizan criterios clínicos muy estrictos. La historia clínica parece que tiende a infravalorar la importancia que la urgencia miccional tiene para la paciente.

La calidad de vida en nuestras pacientes curadas de su incontinencia de esfuerzo está condicionada por la existencia o no de urgencia miccional postoperatoria. Los tres cuestionarios administrados que valoran calidad de vida, (de percepción PGI-S, QoL y reducido ICIQ-SF) han mostrado una gran concordancia entre si, reflejando en sus valores la existencia o no de urgencia miccional. El coeficiente de correlación de Spearman, sin embargo, muestra una correlación solamente ligera, coeficiente 0,376, entre estas dos variables. Creemos que esto expresa una tendencia que se hará mas explicita al ampliar el número de pacientes. Coyne et al. ${ }^{26}$ hallaron también relación inversa entre calidad de vida y urgencia miccional. 
La existencia de urgencia miccional postoperatoria viene condicionada por la existencia de la misma preoperatoriamente, siendo ésta la única variable que ha influido en su aparición, coeficiente de Spearman 0,8.

La calidad de vida afectada no implica que la cirugía no haya sido beneficiosa o no merezca la pena realizarla. No se han administrado cuestionarios preoperatoriamente por lo que no es posible establecer las variaciones de los mismos pero en el cuestionario de impresión global de mejoría (PGI-I) todas las pacientes se encuentran mucho mejor o bastante mejor que antes de operarse.

Dos técnicas han sido publicadas previamente para intentar ajustar la tensión postoperatoriamente. En el REMEX se tensa mediante una polea unos hilos sujetos a un segmento de malla rectangular suburetral. El mayor handicap es precisamente los problemas de dolor e infecciones que la polea situada en hipogastrio puede teóricamente generar. El precio es considerablemente mayor que las mallas habituales.

Cuando se ha usado como primera técnica se han obtenido curaciones de la incontinencia de esfuerzo en el 95\% de los casos, aunque completamente satisfechas solo estaban el 90\% de las pacientes ${ }^{27}$. Posiblemente su uso estaría indicado en aquellos casos reoperados, problemáticos, en los que el éxito depende de una mínima variación de la tensión dada.

El Safyre está formado por una pequeña malla de polipropileno suburetral unida a dos columnas de un polímero de polidilmetilsiloxano, con una configuración tal que impide el descenso de las mismas. El reajuste se puede hacer varios días tras la cirugía y para ello es necesario, con anestesia local o regional, incidir la piel, localizar las columnas y tensar las mismas, todo ello también con incisión quirúrgica vaginal para control de la tensión, situando las tijeras entre la malla y la uretra. Los autores publican un 92\% de éxitos en cuanto a curación de incontinencia se refiere. Comunican una tasa alta de infecciones, el 6\%, habiendo tenido que retirar el $4 \%$ de las mallas, posiblemente debidas a la presencia del polímero ${ }^{28}$.

La técnica de tensión ajustable TVA añade a estas técnicas su sencillez, la no necesidad de incisiones quirúrgicas para el ajuste y la ausencia de potenciales complicaciones, motivadas por la permanencia en el organismo de cuerpos extraños y la composición polimeral de la banda. Los resultados preliminares son prometedores pero habrá que esperar más tiempo de seguimiento, mayor número de casos y la comprobación por otros autores de los mismos antes de llegar a conclusiones definitivas.

\section{REFERENCIAS}

1. Canis Sanchez D, Bielsa Gali O, Cortadillas Angel R, Arango Toro O, Placer Santos J, Gelabert i Mas A. Resultados y complicaciones del TVT en el tratamiento de la incontinencia urinaria de esfuerzo femenina. Actas Urol Esp 2005;29:287-291.

2. Romero Maroto J, Prieto chaparro L, López López C, Guilez Fenoll JM, Bolufer Nadal S. El sling de malla de prolene en el tratamiento de la incontinencia urinaria de esfuerzo. Tratamiento integral de las alteraciones del suelo pélvico. Resultados a largo plazo. Arch Esp Urol 2002;55:1057-1074.

3. Jiménez Calvo J, Hualde Alfaro A, Gonzalez de Garibay AS, Pinós Paul M, Jiménez Arista J, Montesino Semper M, et al. TVT: 3 años de experiencia. Actas Urol Esp 2004;28:13-20.

4. Fritel X, Zabak K, Pigné A, Demaria F, Bénifla JL. Predictive value of urethral mobility before suburethral tape procedure for urinary stress incontinence in women. J Urol 2002;168:2472-2475.

5. Al-Badr A, Ross S, Soroka D, Minassian VA, Karahalios A, Drutz H. Voiding patterns and urodynamics after a tension-free vaginal tape procedure. J Obstet Gynaecol Can 2003;25:725-730.

6. Mazouni C, Karsenty G, Bretelle F, Bladou F, Gamerre M, Serment G. Urinary complications and sexual function after tension-free vaginal tape procedure. Acta Obstet Gynecol Scand 2004;83:955-961.

7. Gateau T, Faramarzi-Roques R, Le Normand L, Glemain P, Buzelin JM, Ballanger P. Clinical and urodynamic repercussions after TVT procedure and how to diminish patients complaints. Eur Urol 2003;44:372-376.

8. Albouy B, Sambuis C, Andreou A, Sibert L, Grise P. Can transobturator tape for urinary incontinence cause complete urinary retention? Prog Urol 2004;14:189-191.

9. deTayrac R, Deffieux X, Droupy S, Chaveaud-Lambling A, Calvanesse-Benamour L, Fernandez H. A prospective randomized trial comparing tension-free vaginal tape and transobturator suburethral tape for surgical treatment of stress urinary incontinence. Am J Obstet Gynecol 2005; 192:339-342.

10. Parick DL, Martin ML, Bushnell DM, Yalcin I, Wagner TH, Buesching DP. Quality of life of women with urinary incontinence: Further development of the incontinence quality of life instrument (I-QOL). Urology 1999;53:71-76.

11. Norton PA, Zinner NR, Yalcin I, Bump RC. Duloxetine versus placebo in the treatment of stress urinary incontinence. Am J Obstet Gynecol 2002;187:40-48.

12. Yalcin I, Bump RC. Validation of two global impression questionnaires for incontinence. Am J Obstet Gynecol 2003;180:98-101.

13. Espuña Pons M, Rebollo Alvarez P, Puig Clota M. Validación de la versión española del Internacional Consultation on Incontinente Questionnaire-Short form. Un cuestionario para evaluar la incontinencia urinaria. Med Clin (Barc) 2004;122:288-292. 
14. Quicios Dorado C, Fernández Fernández E, Gómez García I, Perales Cabanas L, Arias Fuñez F, Escudero Barrilero A. Tratamiento de la incontinencia urinaria de esfuerzo femenina con el sistema TVT: Nuestra experiencia. Actas Urol Esp 2005;29:750-756.

15. Ward KL, Milton P. A prospective multicenter randomized trial of tension-free vaginal tape and colposuspension for primary urodynamic stress incontinente:two-year followup. Am J Obstet Gynecol 2004; 190:324-331.

16. Bumsik H, Sungchan P, Hong SK, and Myung-Soo Ch. Factors predictive of urinary retention after a tension free vaginal tape procedure for female stress urinary incontinente. J Urol 2003;170:852-856.

17. Millar EA, Amundsen CL, Toh KL, Flynn BJ, Webster GD. Preoperative urodynamic evaluation may predict voiding dysfunction in women undergoing pubovaginal sling. J Urol 2003; 169:2234-2237.

18. Nguyen JK, Bhatia NN. Resolution of motor urge incontinente after surgical repair of pelvis organ prolapse. J.Urol 2001;166:2263-2266.

19. Romero Maroto J, Prieto Chaparro L, López López C, Quiles Fenoll JM, Rodríguez E. Obstrucción tras cirugía correctora de la incontinencia femenina de esfuerzo. Diagnóstico y tratamiento. Arch Esp Urol 2002;55:11071114.

20. Abouassaly R, Steimberg JR, Lemieux M, Marois C, Gilchrist LI, Bourque JL, et al. Complications of tensionfree tape surgery: a multi-institutional review. BJU Int 2004;94:110-113.

21. Kaum HJ, Wolf F. TVT: On midurethral tape positioning and its influence on continente Intern. Urogynecol $\mathrm{J}$ 2002; 13:110-115.

22. Lo TS, Wang AC, Horno SG, Liang CC, Soong YK. Ultrasonographic and urodynamic evaluation alter tension free vaginal tape procedure (TVT). Acta Obstet Gynecol Scand 2001;80:65-68.
23. Rodríguez LV, Raz S: Prospective analysis of patients treated with a distal urethral polypropylene sling for symptoms of stress urinary incontinence: Surgical outcome and satisfaction determined by patients driven questionnaires. J Urol 2003;170:857-863.

24. Haab F, Trockman BA, Zimmern PE, Leach GE. Results of pubovaginal sling for the treatment of intrinsic sphincter deficiency determined by questionnaires analysis. J Urol 1997; 158:1738-1741.

25. Chaikin DC, Blaivas JG, Rosenthal JE, Weiss JP. Results of pubovaginal sling for stress incontinence: A prospective comparison of 4 instruments for outcome analysis. J Urol 1999; 162:1670-1673.

26. Coyne KS, Zhou Z, Thompson C, Versi E. The impact on health-related quality of life of stress, urge and mixed urinary incontinence. BJU Int 2003;92:731-735.

27. Sousa-Escandon A, Lema Grille J, Rodriguez Gómez JL, Rios Tallon L, Uribarri González C, Marqués Queimadelos A. Externally readjustable device to regulate sling tensión in stress urinary incontinente: preliminary results. J Endourol 2003;17:515-521.

28. Palma PC, Ricceto CLZ, Dambros M, Thiel M, De Fraga R, Maman JTN, et al. SAFYRE. Un nuevo concepto de cabestrillo ajustable minimamente invasivo para la incontinencia urinaria de estrés femenina. Actas Urol Esp 2004; 10:749-755.

Dr. J. Romero Maroto

Servicio de Urología

Hospital Universitario San Juan de Alicante

Ctra. Alicante-Valencia, s/n

03550 San Juan (Alicante)

E-mail: romero_jes@gva.es

(Trabajo recibido el 5 de diciembre 2005) 